\title{
Emprego de condutos de pericárdio bovino na conexão ventrículo-arterial pulmonar: resultados tardios
}

\author{
Fernando Antônio FANTINI*, Bayard GONTIJO FILHO*, Cristiane MARTINS*, \\ Roberto Max LOPES*, Maria G. HORTA*, Leonardo F. DRUMOND*, Marcelo Frederico de \\ CASTRO*, Carla OLIVEIRA*, Arturo FERRUFINO*, Joăo Alfredo de PAULA E SILVA*, \\ Eduardo PEREDO*, Juscelino Teixeira BARBOSA*, Mário O. VRANDECIC*
}

RBCCV 44205-305

Fantini F A, Gontijo Filho B, Martins C, Lopes R M, Horta M G, Drumond L F, Castro M F, Oliveira C, Ferrufino A, Paula e Silva J A, Peredo E, Barbosa JT, Vrandecic MO. Emprego de condutos de pericárdio bovino na conexâo ventriculo - arterial pulmonar: resultados tardios. Rev Bras Cir Cardiovasc 1996; 11 (3): 161-7.

RESUMO: A reconstrução biventricular de uma série de cardiopatias congênitas requer a interposiçăo de condutos valvados ventriculo-arteriais. Como objetivo de analisar a evoluçăo a longo prazo de um conduto de pericárdio bovino valvulado com bioprótese porcina sem suporte tratado com glutaraldeido, foram revistos os prontuários de 33 pacientes operados de novembro de 1985 a outubro de 1995 . A idade variou de 15 dias a 18 anos (média 5,7 $\pm 4,3$ anos). A atresia pulmonar com comunicaçăo interventricular (CIV) foi a lesāo mais frequeente (16 casos), seguida da sindrome da valva pulmonar ausente (5), truncus arteriosus (4), transposiçāo das grandes artérias com CIV e estenose pulmonar (3) e outras (5). A mortalidade imediata foi de $18,2 \%$, diretamente relacionada à condiçăo pré-operatória. Vinte e três $(70 \%)$ pacientes foram acompanhados por periodos que variaram de 3 meses a 10 anos (média $4,8 \pm 3,0$ anos). A complicaçăo mais frequentemente observada no seguimento tardio foi a estenose da anastomose distal do conduto, presente em $17,4 \%(4 / 23)$ dos pacientes. Foram reoperados $3(13 \%)$ pacientes, sendo que 2 deles por estenose distal $(p=0,02)$ e 1 por endocardite tardia do conduto. A mortalidade tardia foi de $17,4 \%$ (4/23), em 1 caso devido a estenose distal. A causa da estenose distal parece ser devida a retraçăo tecidual na área de transiçăo entre o pericárdio bovino e o tronco pulmonar. Em ate 10 anos de seguimento nāo ocorreu calcificaçăo significativa que prejudicasse a funçāo tanto da valva quanto do conduto. Em conclusão, os condutos de pericárdio bovino apresentaram uma performance satisfatória como substitutos vasculares, nāo tendo ocorrido calcificaçāo significativa da valva porcina ou das paredes do conduto no seguimento tardio. A incide̊ncia de estenose na anastomose distal parece estar mais relacionada a um fenômeno de retraçăo tecidual do que a problemas técnicos.

DESCRITORES: Próteses vasculares. Biopróteses. Ventrículo cardiaco, cirurgia. Artéria pulmonar, cirurgia.

\section{INTRODUÇĀO}

O emprego de condutos extracardíacos tem permitido a correçāo biventricular de diversas cardiopatias congênitas com conexăo ventrículo-arterial pulmonar ausente ou inadequada (1-6). No entanto, - resultado a longo prazo dessa técnica tem sido desapontador, visto que as próteses utilizadas são de material inerte, não apresentam, portanto, crescimento e ainda se degeneram, levando a inevitáveis obstruçōes e a conseqüente necessidade de trocas futuras do conduto $(7-9)$.

Tem sido relatado que os homoenxertos aórtico e pulmonar seriam os condutos de escolha no reestabelecimento da continuidade ventriculo-arte-

Trabalho realizado no Biocór Hospital e na Santa Casa de Misericórdia de Belo Horizonte, MG, Brasil.

Apresentado ao $23^{\circ}$ Congresso Nacional de Cirurgia Cardiaca. Recife, PE, 20 a 23 de marco, 1996.

* Do Biocór Hospital e Santa Casa de Misericórdia de Belo Horizonte.

Endereço para correspondéncia: Fernando A. Fantini. Caixa Postal 106. Beio Horizonte, MG, Brasil. CEP: $30161-970$. 
Fantini F A, Gontijo Filho B, Martins C, Lopes R M, Horta M G, Drumond L F, Castro M F, Oliveira C, Ferrufino A, Paula e Silva J A, Peredo E, Barbosa JT, Vrandecic M O-Emprego de condutos de pericárdio bovino na conexâo ventrículo-arterial pulmonar: resultados tardios. Rev Bras Cir Cardiovasc 1996; 11 (3):161-7.

TABELA 1

CARDIOPATIAS ENCONTRADAS E OBITOS IMEDIATOS RELATIVOS A 33 PACIENTES

\begin{tabular}{|c|c|c|c|c|}
\hline \multirow[t]{2}{*}{ DOENÇA-BÁSICA } & \multicolumn{2}{|c|}{ FREQUUENCIA } & \multicolumn{2}{|c|}{ ÓBITO POI } \\
\hline & $N$. & $\%$ & N. & $\% *$ \\
\hline Atresia Pulmonar com CIV & 16 & 48,6 & 3 & 18.7 \\
\hline Sind. Válva Pulmonar Ausente & 5 & 15,2 & 2 & 40,0 \\
\hline Truncus Arteriosus & 4 & 12,1 & 1 & 25,0 \\
\hline $\mathrm{TGA}+\mathrm{CIV}+\mathrm{EP}$ & 3 & 9.1 & 0 & 0,0 \\
\hline Atresia Puimonar sem CIV & 1 & 3,0 & 0 & 0,0 \\
\hline Discordảncia AV + CIV + EPI & 1 & 3,0 & 0 & 0,0 \\
\hline Dupla Via de Saida do VD + EP & 1 & 3,0 & 0 & 0,0 \\
\hline Dupla Via de Saida do VE + EP & 1 & 3,0 & 0 & 0,0 \\
\hline Endocardite Tardia de Conduto & 1 & 3,0 & 0 & 0,0 \\
\hline Totais & 33 & 100 & 6 & 18.1 \\
\hline
\end{tabular}

$\mathrm{POI}=$ pós-operatório imediato; $\mathrm{TGA}=$ transposiçăo das grandes artérias; $\mathrm{CIV}=$ comunicaçāo interventricular; $\mathrm{EP}=$ estenose pulmonar; $A V=$ atrioventricular; $V D=$ ventriculo direito; $V E=$ ventriculo esquerdo.

"Porcentagem relativa de óbitos

rial (10-11). No entanto, as dificuldades de obtenção, estocagem e, principalmente, de comercializaçāo desses substitutos vasculares praticamente inviabilizam o seu uso nos países em desenvolvimento $(12,13)$. Além disso, em pacientes jovens, $\mathbf{a}$ incidência de degeneração tardia tem sido comparavel à das valvas porcinas $(7,14,15)$

Embora diversas técnicas tenham sido propostas com o intuito de se evitar o uso dessas próteses $(16,17)$, a demanda por condutos valvados ainda é grande. Torna-se, portanto, imperativo o desenvolvimento de produtos de maior durabilidade.

O objetivo do presente trabalho é o de avaliar os resultados a médio e longo prazo de um conduto de pericárdio bovino valvado com bioprótese porcina sem suporte, tratado com glutaraldeído.

\section{CASUISTICA E MÉTODOS}

\section{Pacientes}

No periodo de novembro de 1985 a outubro de 1995, 33 pacientes portadores de cardiopatias com conexão ventrículo-arterial pulmonar ausente ou inadequada foram submetidos a implante de um conduto de pericárdio bovino valvado com bioprótese porcina sem suporte, no Biocór Hospital de Doenças Cardiovasculares e na Santa Casa de Misericórdia de Belo Horizonte. A idade variou de 15 dias a 18 anos (média $5,7 \pm 4,3$ anos) sendo que 5 crianças tinham menos que 1 ano de vida. Quatorze pacientes eram do sexo feminino e 19 do sexo masculino; o peso médio foi de $16,2 \pm 10,8$ quilogramas (variação: 3,0 a $4,9 \mathrm{~kg}$ ) e a área corporal média de $0,7 \pm 0,3$ (variação: 0,2 a 1,4 ).
As lesões abordadas encontram-se relacionadas na Tabela 1. Uma paciente portadora de atresia pulmonar com comunicaçāo interventricular e que havia sido submetida a implante de conduto valvado do tipo Hancock desenvolveu endocardite protética tardia 2 anos após a primeira operação, necessitando, assim, de reoperação. Alteraçōes tronco pulmonar foram identificadas em $33,3 \%$ dos casos, sendo que existia dilataçăo de tronco e artérias pulmonares em 5 pacientes, estenose da artéria direita em 3, estenose da artéria esquerda em 2 e não confluência em 1 paciente. Operaçőes prévias haviam sido realizadas em $18(54,6 \%$ ) pacientes (Tabela 2).

\section{Conduto}

O conduto*1 é composto por um tubo, confeccionado a partir de um retalho de pericárdio bovino com valva porcina selecionada de tamanho adequado, suturada diretamente no interior do tubo, sem qualquer outro suporte senăo uma pequena porção da aorta porcina. $O$ tubo de pericárdio é suturado

\section{TABELA 2}

OPERAÇŌES CARDIACAS PAEVVIAS EM 33 PACIENTES

\begin{tabular}{lc}
\hline OPERAÇÃO & N. DE PACIENTES \\
\hline Blalock-Taussig & 9 \\
Blalock-Taussig Modificado & 5 \\
Waterston & 3 \\
Implante de Conduto Valvado & 1 \\
\hline Total & 18 \\
\hline
\end{tabular}

\footnotetext{
- 'Blocór Indústria e Pesquisas Lıda, Belo Horizonte, Brasil.
} 
Fantini F A, Gontijo Filho B, Martins C, Lopes R M, Horta M G, Drumond L F, Castro M F, Oliveira C, Ferrufino A, Paula e Silva J A, Peredo E, Barbosa JT, Vrandecic M O-Emprego de condutos de pericárdio bovino na conexăo ventrículo-arterial pulmonar: resultados tardios. Rev Bras Cir Cardiovasc 1996;11 (3):161-7.

apenas na sua porçăo média, sendo que as extremidades proximal e distal são deixadas abertas, para que sejam recortadas de acordo com as necessidades individuais de cada caso. A valva é suturada o mais próximo possivel da extremidade distal da sutura do tubo. Os tecidos são afixados em soluçăo tamponada de glutaraldeído e mantidos numa solução de formaldeído. Encontram-se disponiveis em tamanhos variando de $10 \mathrm{~mm}$ a $31 \mathrm{~mm}$ de diâmetro.

\section{TÉCNICA CIRÚRGICA}

Todos os pacientes foram operados através de esternotomia mediana. Uma criança portadora de atresia pulmonar com CIV e grandes colaterais sistêmico-pulmonares recebeu uma toracotomia esquerda prévia no mesmo ato cirúrgico para ligadura das colaterais. Circulação extracorpórea hipotérmica $\left(26^{\circ} \mathrm{C}\right)$ e cardioplegia cristalóide anterógrada gelada foram utilizadas em todos os pacientes. Após ventriculotomia direita, a CIV, quando presente, foi corrigida com remendo de pericárdio bovino. A seleçäo do diâmetro do conduto foi feita levando-se em consideraçăo o calibre do tronco pulmonar e o espaço existente entre o coraçāo e a parede torácica, procurando-se o implante do maior diâmetro possivel. As artérias pulmonares foram tratadas caso apresentassem alguma alteração prévia. A anastomose distal, do tipo término-terminal, foi confeccionada de forma que a valva ficasse o mais próximo possivel dessa anastomose. A anastomose proximai também foi término-terminal, procurando-se um formato do tipo "snake head like" para se diminuir a possibilidade de gradiente.

\section{SEGUIMENTO}

O seguimento dos pacientes foi feito diretamente nos ambulatórios das instituiçōes envolvidas no estudo. Todos os pacientes acompanhados foram submetidos a ecodopplercardiograma seriado e, em caso de alteraçōes de importância, a cateterismo cardíaco de controle. Os dados clínicos e ecocardiográficos tabulados referem-se a visitas ocorridas nos últimos 3 meses.

Esses dados foram estatísticamente analizados através de programa computadorizado (Epi Info versăo 5.01, Epidemiology Program Office, Centers for Disease Control, Atlanta, Geórgia) e sāo apresentados como média mais ou menos um erro padrăo da média). Para comparação das médias foi utilizado o teste $T$ de Student e para comparaçóes dentro dos grupos de variáveis foi aplicada a análise de variância de dados repetidos. Significância estatís- tica foi definida como sendo o valor de $\mathrm{p}$ menor ou igual a 0,05 .

\section{RESULTADOS}

O diâmetro médio dos condutos utilizados foi de $18,6 \pm 3,7$, teoricamente de tamanho adequado para pacientes com área corporal de até 1,5. Procedimentos sobre as artérias pulmonares (AP) foram necessários em $33,3 \%$ dos pacientes, sendo 5 casos de plastia redutota dos ramos pulmonares em pacientes com síndrome da valva pulmonar ausente, 3 casos de plastia de alargamento da artéria pulmonar direita(APD) com remendo de pericárdio bovino, 2 casos de alargamento da esquerda(APE) utilizando-se a própria anastomose distal do conduto e em 1 foi necessária reconstrução da bifurcaçāo do tronco pulmonar.

As complicações ocorridas no pós-operatório imediato (POI), assim como os obitos correspondentes encontram-se na Tabela 3 . Oito pacientes foram reoperados no POI, sendo 1 para drenagem pericárdica, 1 devido a mediastinite, 2 para revisăo de hemostasia e 4 para fechamento secundário do esterno. Este tipo de estratégia foi importante por permitir uma melhor acomodação do conduto no mediastino. A mortalidade imediata foi de $18,2 \%$ (6 pacientes), diretamente relacionada à doença de base e à condição clínica pré-operatória (Tabela 1). Dentre os pacientes reoperados no POI, ocorreram 2 óbitos, sendo um devido a mediastinite e outro por insuficiència respiratória após fechamento secundário do esterno. Cinco pacientes que sofreram procedimentos reconstrutivos do tronco pulmonar foram estudados no POI através de cateterismo cardíaco; em todos, a correção mostrou-se adequada.

Dos 27 pacientes sobreviventes, năo foi possível obter informaçōes adequadas de 4 casos, o que resultou em uma taxa de seguimento de $70 \%$. 0 tempo de observaçăo variou de 3 meses a 10 anos, com média de $4,8 \pm 3,0$ anos.

TABELA 3

COMPLICACÖES DO PÓS-OPERATÓRIO IMEDIATOE RESPECTIVOS ÓBITOS EM 33 PACIENTES

\begin{tabular}{lccc}
\hline COMPLICAÇŌES & \multicolumn{2}{c}{ FREQŨENCIA } & OBITOS \\
& N. & $\%$ & N. \\
\hline Sindrome de Baixo Débito & 6 & 18,1 & 4 \\
Hemorragia & 2 & 6,1 & 0 \\
Insuficiència Respiratória & 2 & 6,1 & 1 \\
Mediastinite & 1 & 3,0 & 1 \\
Derrame Pericárdico & 1 & 3,0 & 0 \\
\hline Totais & 12 & 36,3 & 6 \\
\hline
\end{tabular}


Fantini F A, Gontijo Filho B, Martins C. Lopes R M, Horta M G, Drumond L F, Castro M F, Oliveira C, Ferrufino A, Paula e Silva J A, Peredo E, Barbosa JT, Vrandecic M O - Emprego de condutos de pericárdio bovino na conexáo ventrículo-arterial pulmonar: resultados tardios. Rev Bras Cir Cardiovasc 1996; 11 (3):161-7.

Os dados ecodopplercardiográficos relacionados ao conduto obtidos nos 23 pacientes acompanhados estăo relacionados na Tabela 4. Na maioria dos casos o gradiente encontrado não era hemodinamicamente significativo. No entanto, em 4 pacientes, gradientes superiores a $60 \mathrm{mmHg}$ puderam ser detectados. Insuficiência da valva do conduto foi vista em apenas 2 pacientes, sendo que 1 deles era portador de doença vascular pulmonar importante.

Nove pacientes com alteraçōes de importância clínica ou ecocardiográfica foram submetidos a cateterismo cardiaco de controle, num periodo de tempo que variou de 10,8 meses a 8,6 anos (Tabela 5). A complicação mais frequiente foi a estenose na anastomose distal do conduto, presente em 3 dos pacientes; foi ainda encontrado: desproporção entre o tamanho do conduto e a área corporal do paciente, insuficiência ventricular direita e hipertensăo pulmonar sem alteraçăo da funçāo do conduto e 2 pacientes com CIV residual sem importáncia hemodinâmica. Em 1 caso, nenhuma, alteração foi evidenciada.

Tanto na radiografia convencional de tórax. quanto nos exames complementares acima citados, não foi possivel a detecção de calcificaçăo de importância na valva ou nas paredes do conduto.

Reoperaçōes tardias após 11,5 meses a 4,5 anos foram necessárias em $3(13 \%)$ pacientes. Em 1 deles o conduto foi trocado devido à presença de endocardite tardia na prótese. Nos outros dois, a indicação cirúrgica deveu-se a estenose na anastomose distal $(p=0,02)$. Na reoperaçăo, apresentavam fibrose intensa ao redor do conduto, com perda dos planos de clivagem e retraçāo cicatricial ao nivel da anastomose da prótese com o tronco pulmonar. Em 1 caso o conduto foi trocado e em outro foi realizada plastia de alargamento da área estenosada. Dois pacientes aguardam reoperaçăo, sendo 1 por estenose da anastomose distal e outro por desproporçāo paciente-conduto.

A mortalidade tardia foi de $17,4 \%$ (4/23), tendo ocorrido de 3 meses a 4,5 anos após a operaçăo. Uma criança morreu subitamente, sem qualquer evidência de problema com relaçăo ao conduto. Em outro caso, o óbito foi devido a septicemia, 3 meses após a troca do conduto, por endocardite. Um dos pacientes reoperados faleceu na sala de operaçōes, por sangramento incontrolável. O último óbito ocorreu em uma criança portadora de sindrome da valva pulmonar ausente, submetida a plastia redutora das artérias pulmonares. O cateterismo cardiaco de controle realizado no $\mathrm{POI}$ mostrou adequada redução do diâmetro das artérias pulmonares e ausência de gradiente ao nivel das anastomoses. Ecocardiograma realizado no $4^{\circ}$ mês de pós-operatório denotou a presença de gradiente na anastomose
TABELA 4

AESULTADO TARDIO - ACHADOS ECODOPPLERCARDIOGRAFICOS EM 23 PACIENTES

\begin{tabular}{llrr}
\hline & & N. & $\%$ \\
\hline \multirow{3}{*}{ Gradiente } & $<20 \mathrm{mmHg}$ & 14 & 60,8 \\
& $21-36 \mathrm{mmHg}$ & 4 & 17,4 \\
& $36-60 \mathrm{mmHg}$ & 1 & 4,3 \\
& $>60 \mathrm{mmHg}$ & 4 & 17,4 \\
Regurgitação & Ausente & 21 & 91,4 \\
& Leve & 1 & 4,3 \\
Vegetaçōes & & 1 & 4,3 \\
& & 1 & 4,3 \\
\hline
\end{tabular}

distal, com discreta estenose a este nivel. Esta criança retornou ao hospital no $11 \%$ mês de PO em franca insuficiência ventricular direita e faleceu antes que qualquer medida pudesse ser tomada. A necropsia realizada mostrou os mesmos achados de intensa retração cicatricial na área de transiçāo entre o pericárdio bovino e o tronco pulmonar. Os cortes histológicos das peças cirúrgicas $\theta$ de necropsia mostram, em geral, o tubo de pericárdio envolvido externamente por proliferaçăo fibrovascular, com moderado infiltrado inflamatório predominantemente mononuclear. Internamente, nota-se camada mais espessa de tecido fibroblástico e hialinizado, com poucas células inflamatórias. As válvulas săo parcialmente revestidas por material amorfo, contendo histiócitos e células grânulo-mononucleares. Céluias endoteliais não são identificadas. Em peças mais antigas, discretos focos de calcificaçăo no pericárdio bovino podem ser identificados.

\section{COMENTÁRIOS}

As dificuldades de manutençăo de bancos de homoenxertos, em nosso meio, assim como os maus resultados relatados com próteses sintéticas na conexāo entre o coraçäo e o tronco pulmonar $(8,18)$ proporcionaram a investigaçăo de outras opçōes de materiais. O pericárdio bovino tratado com glutaraldeído, em decorrência de suas características (19), tem sido largamente usado em diversas circunstân-

TABELA 5

CATETERISMO CARDIACO EM 9 PACIENTES

\begin{tabular}{lc}
\hline ACHADOS & N. \\
\hline Estenose Anastomose Distal & 3 \\
Desproporçăo Conduto-Paciente & 1 \\
Insuficiência Ventricular Direita & 1 \\
Hipertensåo Pulmonar & 1 \\
CIV Residual & 2 \\
Sem Alteraçōes & 1 \\
\hline
\end{tabular}


Fantini F A, Gontijo Filho B, Martins C, Lopes R M, Horta M G, Drumond L F, Castro M F, Oliveira C, Ferrufino A, Paula e Silva J A, Peredo E, Barbosa JT, Vrandecic MO - Emprego de condutos de pericárdio bovino na conexão ventrículo-arterial pulmonar: resultados tardios. Rev Bras Cir Cardiovasc 1996; 11 (3):161-7.

cias, tanto para correçōes intracardiacas ${ }^{(20)}$, como dos grandes vasos da base (21,22). Ele se aproxima bastante do tecido ideal, por ser liso, flexivel, de paredes finas, portanto de fácil manejo e bastante hemostático. Além disso, é de baixo custo e facilmente obtido e estocado.

Vários tipos de conduto de pericárdio têm sido utilizados em nosso meio $(18,23,24)$. O conduto que vem sendo desenvolvido pelo nosso grupo mostra um enxerto de pericárdio bovino suturado de forma a perfazer a funçäo tubular, no interior do qual é incorporada uma valva porcina sem suporte. Segue, portanto, a filosofia do nosso grupo com relaçăo aos xenoenxertos, o conceito "stentless" (25), com dese. nhos o mais próximo do natural para năo causar turbulência. Na presente série, o comportamento da valva porcina tem sido excelente e, mesmo no seguimento mais tardio, ela continua funcionando adequadamente, sem causar obstruçăo no interior do conduto.

No entanto, os tecidos biológicos de uma ma. neira geral se degeneram com o passar do tempo independentemente do local implantado. Segundo GABBAY et al. (26) realizaram, em cāes, implante de pericárdio bovino tratado com glutaraldeido em diferentes locais do coração; quando implantado na parede atrial esquerda, o pericárdio mostrava-se invariavelmente espessado $\theta$, principalmente, retraido, com intensa reação fibrótica e calcificaçăo. Na substituição de cordas terndineas, apesar de espessado e calcificado, mantinha as suas dimensōes originais. Essas observações são de fundamental importância, pois comprovam a resistência do pericárdio ao estresse físico, na forma de pressão ou tensăo e corroboram os resultados que têm sido obtidos com esse material. Em vasos de alta pressāo, como a aorta, o pericárdio apresenta excelente performance a curto e longo prazo $(27,28)$, em locais como o tronco pulmonar o pericárdio tende a se retrair, podendo levar a estenose $(29,30)$. Assim sendo, a presença de uma anastomose término-terminal de um tecido que sofre retração numa câmara de baixa pressăo explica os achados de estenose na área de transiçăo entre o tronco pulmonar e o conduto val- vado observado na nossa série. Portanto, a inclusăo de um "stent" próximo à extremidade distal do conduto deve diminuir a incidência de complicaçōes a esse nivel.

Estudos recentes sugerem que o pericárdio bovino tratado com glutaraldeido pode induzir resposta imunológica in vivo, consistente com uma reaçăo do tipo hospedeiro-versus-prótese (31). Este tipo de resposta 6 também observado em outros tecidos biológicos (32,33). Estudos laboratoriais e clínicos em andamento na nossa Instituiçäo ${ }^{(25)} \mathrm{com}$ um novo método de tratamento anticalcificante (No React ${ }^{\mathrm{T} M}$ ) têm conseguido tecidos biológicos $100 \%$ citocompativeis, que não produzem reação de corpo estranho. Além de provocar menor grau de calcificação, a superfície dos enxertos permite a formaçāo de neoíntima verdadeira, como pode ser observado em estudos imuno-histoquimicos para demonstraçăo do fator 8 (marcador de células endoteliais) realizados em peças recuperadas. Embora a degeneração dos tecidos nāo tenha constituído problema na presente casuística, já que o grau de espessamento e calcificação encontrado tanto no tubo de pericárdio, quanto na valva porcina não prejudicaram o funcionamento do conduto, a utilização de tecidos totalmente histocompatíveis deve determinar maior longevidade aos heteroenxertos, melhorando, assim, os resultados a longo prazo.

Obviamente, os resultados tardios obtidos com os diversos tipos de condutos estão longe do ideal $(34,35)$ e os números da presente série não fogem a essa regra. No entanto, as observações extraídas desse grupo de pacientes nos permitem concluir que: a) o pericárdio bovino comprovou ser um bom substituto vascular, perfazendo de maneira satisfatória a funçāo tubular a que se destina; b) a calcificação da valva porcina ou das paredes do conduto não foi significativa, năo tendo se constituido, dessa forma, em fator de mau prognóstico; c) houve uma incidência aumentada de estenoses na transição entre o pericárdio bovino e - tronco pulmonar, que parece estar relacionada a um fenômeno de retraçāo tecidual de caráter cicatricial. 
Fantini F A Gontijo Filho B, Martins C, Lopes R M, Horta M G, Drumond L F, Castro M F, Oliveira C, Ferrufino A, Paula e Silva J A, Peredo E, Barbosa JT, Vrandecic M O-Emprego de condutos de pericárdio bovino na conexăo ventriculo-arterial pulmonar: resultados tardios. Rev Bras Cir Cardiovasc 1996; 11 (3):161-7.

\section{RBCCV 44205-305}

Fantini F A, Gontijo Filho B, Martins C, Lopes R M, Horta M G, Drumond L F, Castro M F, Oliveira C, Ferrufino A, Paula e Silva J A. Peredo M, Barbosa JT, Vrandecic M O-Late results with the use of a valved conduit of bovine pericardium for ventricle to pulmonary artery connection. Rev Bras Cir Cardiovasc1996:11 (3): $161-7$.

ABSTRACT: The biventricular repair of congenital heart diseases with an inadequate or absent ventricle to pulmonary artery connection sometimes requires the use of extracardiac conduits. In order to study the longterm outcome of a glutaraldehyde-preserved bovine pericardial conduit valved with a stentless porcine aortic valve, we reviewed the data of 33 patients operated between November 1985 and October 1995 . Patients ages ranged from 15 days to 18 years (mean $5.7 \pm 4.3$ years). Pulmonary atresia with ventricular septal defect (VSD) was the most frequent disease (16 cases), followed by tetralogy of Fallot with absent pulmonary valve (5), truncus arteriosus (4), transposition of great arterios with VSD and pulmonary stenosis (3) and other miscellaneous (5). The overall hospital mortality was $18.2 \%$ and was related to the preoperative clinical condition; 23 patients $(70 \%$ ) were followed by a mean of $4.8 \pm 3.0$ years (ranging from 3 months to 10 years). The most common late complication observed was stenosis of the anastomosis between the conduit and the pulmonary artery present in $17.4 \%(4 / 23)$ of the patients and was the cause of 2 later reoperations $(p=0,02)$; another patient was reoperated for late conduit endocarditis. This complication was also the cause of 1 of the 4 late deaths $(17.4 \%)$. The last patient is waiting for surgery. The development of the anastomotic stenosis was problably due to a retraction of the pericardial tissue along the transition with the thin wall of the pulmonary artery. Up to 10 years, gross calcification impairing the function of the valve or the conduit itself could not be detected. In conclusion, the bovine pericardial conduits have shown a good performance as a vascular substitute. Calcification has not been a major drawback. The righ incidence of distal stenosis appears to be more related to a shrinking of the pericardial tissue than to technical reasons. surgery.

DESCRIPTORS: Vascular prostheses. Bioprostheses. Heart ventricle, surgery. Puimonary artery.

\section{REFERÊNCIAS BIBLIOGRÁFICAS}

1 Kirklin J W \& Barrat-Boyes B G - Cardiac surgery. New York: Churchill Livingstone, 1988: $758 \mathrm{p}$.

2 Klinner W \& Zenker R - Experience with correction of Fallot's tetralogy in 178 cases. Surgery 1961; 57 : 353-7.

3 McGoon D C, Rastelli G C, Ongley P A - An operation for the correction of truncus arterious. JAMA 1968: 205: 69-73.

4 Rastelli G C, Ongley P A, Davis G D, Kirklin J W. Surgical repair for pulmonary valve atresia with coronary-pulmonary artery failure: report of a case. Mayo Clin Proc 1965; 40: 521-7.

5 Rastelli G C, Wallace R B, Ongley P A - Complete repair of transposition of the great arteries with pulmonary stenosis: a review and report of a case corrected by using a new surgical technique. Circulation 1969; 39: 83-95.

6 Ross D N \& Somerville J - Correction of pulmonary atresia with a homograft aortic valve. Lancet 1966;2: 1446-7.

7 Bull C, Macartney F J, Horvath P - Evalution of longterm results of homograft and heterograft valves in extracardiac conduits. J Thorac Cardiovasc Surg 1987: $94: 12-19$.
8 Jonas R A, Freed M D, Mayer J E, Castañeda A R . Long-term follow-up of patients with synthetic right heart conduits. Circulation 1985; 72 (Suppl.2): 77. 83.

9 Razzouk A J, Williams W G, Cleveland D C et al. Surgical connections from ventricle to pulmonary artery: comparison of four types of valved implants. Circulation 1992; 86 (Suppl.2): 154-8.

10 Bando K, Danielson G K, Schaft H V, Mair D D, Julsrud P R, Puga F P - Outcome of pulmonary and aortic homografts for right ventricular outflow tract reconstruction. J Thorac Cardiovasc Surg 1995; 109: 509-18.

11 Kay P H \& Ross D N - Fifteen year's experience with the aortic homograft: the conduit of choise for right ventricular outflow tract reconstruction. Ann Thorac Surg 1985; 40: 360-4.

12 Barbero-Marcial M, Baucia J A, Jatene A - Valved conduits of bovine pericardium for right ventricle to pulmonary artery connections. Semin Thorac Cardiovasc Surg 1995; 7: 148-53.

13 lyer K S \& Sharma R - The right ventricule to pulmonary artery connection: when homografts are not always available. Semin Thorac Cardiovasc Surg 1995; 7 : 145-7. 
Fantini F A, Gontijo Filho B, Martins C, Lopes R M, Horta M G, Drumond L F, Castro M F, Oliveira C, Ferrufino A, Paula e Silva J A, Peredo E, Barbosa JT, Vrandecic MO - Emprego de condutos de pericárdio bovino na conexäo ventriculo-arterial pulmonar: resultados tardios. Rev Bras Cir Cardiovasc 1996; 11 (3):161-7.

Cleveland D C, Williams W G, Razzouk A J - Failure of cryopreserved homograft valved conduits in the pulmonary circulation. Circulation 1992; 86 (Suppl.2): 150-3.

15 Sano S, Karl T R, Mee R B B - Extracardiac valved conduits in the pulmonary circuit. Ann Thorac Surg 1991; 52: $285-90$.

16 Barbero-Marcial M, Riso A, Atik E, Jatene A - A technique for correction of truncus arteriosus types I and II without extracardiac conduits. J Thorac Cardiovasc Surg 1990; 99: 364-9.

17 Lecompte Y, Neveux JY, Leca F et al. - Reconstruction of the pulmonary outflow tract without prosthetic conduit. J Thorac Cardiovasc Surg 1982; 84: 727 33.

18 Kobayashi J, Backer C L, Zales V R, Crawford S E, Muster A J, Mavroudis C - Failure of hemashield extension in right ventricle-to-pulmonary artery conduits. Ann Thorac Surg 1993; 56: 277-81.

19 Ishihara T, Ferrans V J, Jones M, Boyce S W, Roberts W C - Structure of bovine parietal pericardium and of unimplanted Ionescu-Shiley pericardial valvular bioprostheses. J Thorac Cardiovasc Surg 1981; 81: $747-57$.

20 De Leval M - Ventricular septal defects. In: Stark J \& De Leval M - Surgery for congenital heart defects. 2.ed. Philadelphia W. B. Sauders, 1994: 355-71.

21 Fantini F A, Gontijo Filho B, Vrandecic M O et al. Substítuiçāo da aorta ascedente e arco ab́rtico por enxerto de pericárdio bovino: resultados a médio prazo. Rev Bras Cir Cardiovasc 1994; 9: 81-7.

Gontijo Filho B, Fantini F A, Silva J A P et al. - Correção cirúrgica da estenose supra-aórtica localizada: relato de 2 casos. Arq Bras Cardiol 1984; 43: 433-6

Moraes C R, Rodrigues J V. Gomes C A et al. - Operaçáo de Rastelli utilizando-se conduto valvulado de pericárdio bovino: experiência inicial. Rev Bras Cir Cardiovasc 1988; 3: 84-92.

24 Santos J L V, Braile D M, Soares M J F et al - Avaliaçăo de tubo valvulado de pericárdio bovino em modelo experimental animal. Rev Bras Cir Cardiovasc 1990 5: $16-25$
25 Vrandecic M, Gontijo B, Fantini F, Gabbay S, Vrandecic $E$ A, Vrandecic E - The stentless heart valve concept. New anticalcificant tissue tanning: pre-clinical trial. In: Piwnica A \& Westaby S, eds. Stentless bioprostheses. Oxford: Isis Medical Media, 1990: 93-9.

26 Gabbay S, Bortolotti U, Factors S, Shore D F, Frater M $B$ - Calcification of implanted xenograft pericardium: influence of site and function. $J$ Thorac Cardiovasc Surg 1984; 87: 782-7.

27 Braile D M, Ardito R V, Greco O T - Alargamento da raiz da aorta com "patch" de pericárdio bovino preservado pelo glutaraldeido. Arq Bras Cardiol 1983; 41 : 289-96.

28 Gontijo B, Vrandecic M, Fantini F et al. - Replacement of the ascending aorta and aortic arch with bovine pericardial grafts. Eur J Cardio-thorac Surg 1995: 9: $127-32$.

29 Fantini F A, Gontijo Filho B, Lopes R M - Cirurgia de Glenn bidirecional: importância da manutençăo de fluxo "pulsátil" na artéria pulmonar. Rev Bras Cir Cardiovasc 1995; 10: 25-33.

30 Lamberti J J, Spicer R L, Waldman J D et al. - The bidirecional cavopulmonary shunt. J Thorac Cardiovasc Surg 1990; 100; 22-30.

31 Dahm M, Lyman W D, Schwell A B, Frater R W M. Imuno-genicity of glutaraldehyde tanned bovine pericardium. J Thorac Cardiovasc Surg 1990; 99 $1082-90$

32 Imai $Y$, Takanashi $Y$, Hoshino S, Nakata S - The equine pericardial valved conduit and current strategies for pulmonary reconstruction. Semin Thorac Cardiovasc Surg 1995; 7: 157-61.

33 Rocchini A P, Weesner K M, Heildelberger K, Keren D, Behrendt $D$, Rosenthal A - Porcine xenograft valve failure in children: an immunologic response. Circulation 1981; 64 (Suppl.2): 162-71.

34 Danielson G K, Anderson B J, Scheleck C D, IIstrup D $M$ - Late results of pulmonary ventricle to pulmonary artery conduits. Semin Thorac Cardiovasc Surg 1995; 7: 162-7.

35 Salles C A, Riboiro N A M, Kalil R A et al. - Reconstruçăo arterial com tubo de pericárdio bovino corrugado. Rev Bras Cir Cardiovasc 1992; 7: 88-95. 